\section{Bronchiectasis in bone marrow transplantation}

\author{
Richard Scott Morehead
}

\begin{abstract}
Two patients are described with clinical and radiographic bronchiectasis which occurred after allogeneic bone marrow transplantation for haematological malignancy. Both had evidence of chronic graft versus host disease in other organs. Increased immunosuppression with corticosteroids resulted in clinical response, although both patients persisted with chronic mucopurulent sputum production and one had progressive airflow obstruction. Bronchiectasis may be an underrecognised manifestation of chronic graft versus host disease of the lung.

(Thorax 1997;52:392-393)
\end{abstract}

Keywords: bone marrow transplantation, bronchiectasis, graft versus host disease.

Pulmonary complications following bone marrow transplantation are common and include pulmonary oedema, infectious pneumonia, interstitial pneumonitis, alveolar haemorrhage, and bronchiolitis obliterans. In this population airflow obstruction usually represents bronchiolitis obliterans and occurs in approximately $10 \%$ of patients following bone marrow transplantation with chronic graft versus host disease (GVHD). ${ }^{12}$ Bronchiectasis has rarely been reported in association with bone marrow transplantation. ${ }^{3-6}$ Although the presenting symptoms of bronchiolitis obliterans are usually dyspnoea and dry cough, case series document patients that produce copious sputum reminiscent of bronchiectasis. ${ }^{7}$ The limitations of chest radiology in the diagnosis of bronchiolitis obliterans and bronchiectasis are well known; however, high resolution computed tomographic (HRCT) scanning is sensitive in detecting bronchial dilatation. ${ }^{8}$ We report two previously normal patients seen following bone marrow transplantation with recurrent sinopulmonary infections and evidence of bronchiectasis on the HRCT scan.

\section{Case reports}

PATIENT 1

A 37 year old white man was evaluated for recurrent sinopulmonary infections and an abnormal chest radiograph. His past history was remarkable for allogeneic bone marrow transplantation 65 months previously for a myeloproliferative disorder. Apart from a pleural effusion during initial therapy which resolved, there was no pulmonary disease before transplantation and pulmonary function testing revealed moderate restriction with a forced expiratory volume in one second $\left(\mathrm{FEV}_{1}\right)$ of 2.54 1 (54\% predicted), forced vital capacity (FVC) of 3.021 ( $53 \%$ predicted), $\mathrm{FEV}_{1} / \mathrm{FVC}$ of 0.84 , total lung capacity (TLC) of 3.971 (54\% predicted), and diffusion capacity for carbon monoxide corrected for alveolar volume and haemoglobin (TLCO/VA) of $105 \%$ predicted. He subsequently developed chronic GVHD with skin, eye, mucosal, and gastrointestinal involvement. Over the ensuing five years he experienced recurring bouts of bronchospasm, mucopurulent sputum production, and sinusitis requiring increasing doses of corticosteroids, intravenous antibiotics, and several sinus drainage procedures. Repeat lung function testing after treatment showed normal levels of TLC of 7.201 (98\% predicted) with normal airflow and diffusion.

On this admission the chest radiograph revealed a left lower lobe abnormality, and sinus films demonstrated mucosal thickening without air-fluid levels. HRCT scanning revealed parenchymal nodules with bronchiectasis (fig 1). Pulmonary function tests demonstrated normal airflow and restriction with a TLC of 4.391 (60\% predicted) and TLCo/VA of $111 \%$ predicted. Bronchoscopy revealed purulent secretions and transbronchial biopsy specimens showed bronchial inflammation. Respiratory cultures grew normal flora and were negative for other pathogens. Quantitative immunoglobins (Ig) were normal except for reduced IgG subclass 4. Symptoms responded with corticosteroids and intravenous antibiotics and he was discharged from the hospital. After discharge he was lost to follow up.

\section{PATIENT 2}

A 44 year old white woman was admitted for dyspnoea and pleuritic chest pain of acute onset corresponding to a left lower lobe infiltrate. Her past history was significant for an allogeneic bone marrow transplantation 13 months previously for acute myelogenous leukaemia. This was complicated during the first 100 days by chronic GVHD with eye and gastrointestinal involvement. Concurrently, symptoms of sinusitis and purulent bronchitis

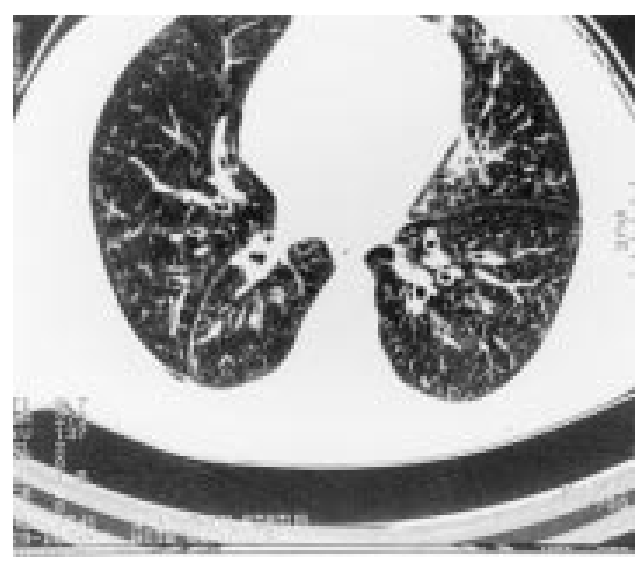

Figure 1 Thoracic HRCT scan of patient 1 revealing bilateral bronchiectasis mainly in lower lobes. 
necessitated several hospital admissions for intravenous antibiotics, whereas evaluation before the bone marrow transplantation was negative for pulmonary disease with normal pulmonary function tests. Testing 100 days after the bone marrow transplantation showed pulmonary restriction with $\mathrm{FEV}_{1}$ of $2.271(79 \%$ predicted), $\mathrm{FEV}_{1} / \mathrm{FVC}$ of 0.84 , TLC of 3.671 ( $73 \%$ predicted), and TLCO/VA of $78 \%$ predicted. Bronchoscopy with transbronchial biopsy specimens was unrevealing at that time.

On this admission evaluation for venous thromboembolism was negative. An HRCT scan revealed parenchymal nodules and bronchiectasis, and pulmonary function tests now demonstrated combined restrictive and obstructive impairment with $\mathrm{FEV}_{1}$ of $1.191(42 \%$ predicted), FVC of $1.931, \mathrm{FEV}_{1} / \mathrm{FVC}$ of 0.62 (bronchodilator response unassessed), TLC of 3.411 (67\% predicted), and TLCo/VA of $91 \%$ predicted. Bronchoscopy with transbronchial biopsy specimens revealed an inflammatory infiltrate involving alveoli and airway epithelium, and thoracoscopic biopsy showed bronchiolitis and neutrophilic inflammation. A single strongyloides organism was identified in an uninvolved area. All stool, sputum, and bronchoscopic samples were negative for larval forms. Treatment with thiabendazole, corticosteroids, and antibacterial agents yielded a marked decrease in dyspnoea, cough, and sputum production. However, clinical deterioration occurred three weeks after rapid tapering of corticosteroids. HRCT scanning confirmed progression of nodules and continued bronchiectasis, and repeat transbronchial biopsy specimens revealed organising pneumonia with severe bronchial inflammation. Sputum and bronchoscopic samples grew Haemophilus influenzae but were negative for strongyloides. Reinstitution of corticosteroids resulted in an improvement in symptoms, radiographic features, and pulmonary function tests with continued airflow obstruction: $\mathrm{FEV}_{1} 1.37$ 1, FVC 2.251 , and $\mathrm{FEV}_{1} /$ FVC of 0.61 .

She has been maintained on cyclosporin and prednisone with clear radiographs but has recurrent bouts of mucopurulent sputum production and progressive airflow obstruction. Twice during periods of clinical stability low IgA levels at 65 and $75 \mathrm{mg} / \mathrm{dl}$ (normal 100-420) have been found with normal IgM and IgG levels (both total and subclasses). The sweat chloride level was $14 \mathrm{mmol} / 1$ (normal 5-45). Thirty months after bone marrow transplantation she is oxygen dependent with an $\mathrm{FEV}_{1}$ of 0.881 .

\section{Discussion}

These two cases demonstrate recurrent sinopulmonary infection due to bronchitis following allogeneic bone marrow transplantation. Case 1 has had normal airflow obstruction during a five year follow up and case 2 has persistent airflow obstruction despite initial improvement. As sinusitis coexisted, it is possible to implicate seeding of the lower respiratory tract as the primary factor in the development of bronchiectasis. However, the temporal relationship between transplantation and disease onset, the absence of prior symptoms or pulmonary function abnormalities, the improvement with augmented immunosuppression, and the inability to elucidate another cause suggest chronic GVHD as the likely aetiology of their pulmonary condition.

Bronchiectasis has been increasingly recognised following bone marrow transplantation..$^{3-5}$ Philit et al described four bone marrow transplantation patients with chronic GVHD and obstructive lung disease who had evidence of bronchiectasis on the HRCT scan; two of these had biopsy proven bronchiolitis obliterans. ${ }^{6}$ To date, all reports of bronchiectasis following bone marrow transplantation have been retrospective and have involved symptomatic patients. Because HRCT scanning is a relatively new technique, bronchiectasis may be more common than is generally perceived. Seemingly, this is the case in lung transplantation where bronchiectasis by HRCT scanning frequently coexists with bronchiolitis obliterans related to chronic rejection..$^{910}$ Wider use of HRCT scanning may be useful in identifying chronic GVHD involving the lung.

1 Chan CK, Hyland RH, Hutcheon MA, Minden MD, Alexander MA, Kossakowska AK, et al. Small-airways disease in recipients of allogeneic bone marrow transplants. Medicine 1987;66:327-40.

2 Clark JG, Crawford SW, Madtes DK, Sullivan KM. Obstructive lung disease after allogeneic marrow transplantation. Ann Intern Med 1989;111:368-76.

3 Graham NJ, Muller NL, Miller RR, Shepherd JD. Intrathoracic complications following allogeneic bone marrow transplantation: CT findings. Radiology 1991;181: row trans-6.

4 Sargent MA, Cairns RA, Murdoch MJ, Nadel HR, Wensley D, Schultz KR. Obstructive lung disease in children after bone marrow transplantation: evaluation with high resolution CT. AfR 1995;164:693-6.

5 Paz HL, Crilley P, Patchefsky A, Schiffiman RL, Brodsky I. Bronchiolitis obliterans after autologous bone marrow I. Bronchiolitis obliterans after autolog
transplantation. Chest 1992;101:775-8.

transplantation. Chest 1992;101:775-8.
6 Philit F, Weisendanger T, Archimbaud E, Mornex JF, Brune J, Cordier JF. Post-transplant obstructive lung disease ("bronchiolitis obliterans"): a clinical comparative study of bone marrow and lung transplant patients. Eur Respir f 1995;8:551-8.

7 Ralph DD, Springmeyer SC, Sullivan KM, Hackman RC, Storb R, Thomas ED. Rapidly progressive air-flow obstruction in marrow transplant recipients. Am Rev Respir Dis 1984;129:641-4.

8 Grenier P, Maurice F, Musset D, Menu Y, Nahum H. Bronchiectasis: assessment by thin section CT. Radiology 1986;161:95-9.

9 Morrish WF, Herman SJ, Weisbrod GL, Chamberlain DW. Bronchiolitis obliterans after lung transplantation: findings at chest radiography and high-resolution CT. Radiology 1991;179:487-90.

10 Loubeyre P, Revel D, Delignette A, Wiesendanger T, Philit $\mathrm{F}$, Bertocchi M, et al. Bronchiectasis detected with thinsection CT as a predictor of chronic lung allograft rejection. Radiology 1995;194:213-6. 